\title{
A Systematic Synthesis Framework for Extractive Distillation Processes
}

\author{
S. Kossack ${ }^{\mathrm{a}}$, K. Kraemer ${ }^{\mathrm{a}}$, R. Gani ${ }^{\mathrm{b}}$, W. Marquardt ${ }^{\mathrm{a}}$ \\ ${ }^{a}$ Process Systems Engineering, RWTH Aachen University, D-52064 Aachen, Germany \\ ${ }^{b}$ Department of Chemical Engineering, Technical University of Denmark, DK-2800 Lyngby, Denmark
}

\begin{abstract}
The effectiveness of an extractive distillation process relies on the choice of the extractive agent. In this contribution heuristic rules for entrainer selection and for the design of entrainers through computer-aided molecular design are reviewed first. The potential of the generated alternatives is then evaluated by their selectivity at infinite dilution and by the rectification body method (RBM). It is shown that a screening based on selectivity alone is not sufficient and could possibly lead to an unfavorable entrainer choice. The minimum entrainer flowrate and the minimum energy demand, calculated from the RBM, allow a more comprehensive evaluation of different entrainer alternatives. In a third step a rigorous MINLP optimization of the entire extractive flowsheet for the remaining entrainer candidates is executed to fix the remaining design degrees of freedom and to determine the best entrainer. Since a number of alternative entrainers have already been eliminated, only a few optimization runs are necessary. These steps form a framework which facilitates the systematic generation and evaluation of entrainer alternatives. The suggested synthesis framework is illustrated by a case study for the separation of acetone and methanol.
\end{abstract}

Keywords: extractive distillation, entrainer selection, CAMD, RBM, MINLP

\section{Extractive distillation}

Separation of close-boiling or even azeotropic mixtures in a single simple distillation column is usually not feasible. The first step in the conceptual design of a distillation process for such a non-ideal mixture is the evaluation of the pressure sensitivity of the azeotrope (5). If the azeotropic composition is insensitive to pressure change, then the addition of another component, the so-called entrainer ${ }^{1}$, can be used to alter the relative volatility of the close-boiling or azeotrope forming components. This entrainer is selected to facilitate the separation in a distillation process.

Doherty and Malone (5) classify possible entrainer candidates into four categories:

1. liquid entrainers that do not induce liquid-phase separation in the ternary mixture,

\footnotetext{
${ }^{1}$ Other names include solvent or (mass) separating agent (5).
} 
2. liquid entrainers that induce liquid-phase separation in the ternary mixture,

3. entrainers that react with one of the components in the mixture, and

4. entrainers that ionically dissociate in the original mixture and alter the VLE.

In this paper, we focus on entrainers of the first category, a distillation type that is also referred to as homogeneous azeotropic distillation. Here, the entrainer influences the distribution of the components in the vapor and liquid phases by interacting with one or more of the components in the liquid phase.

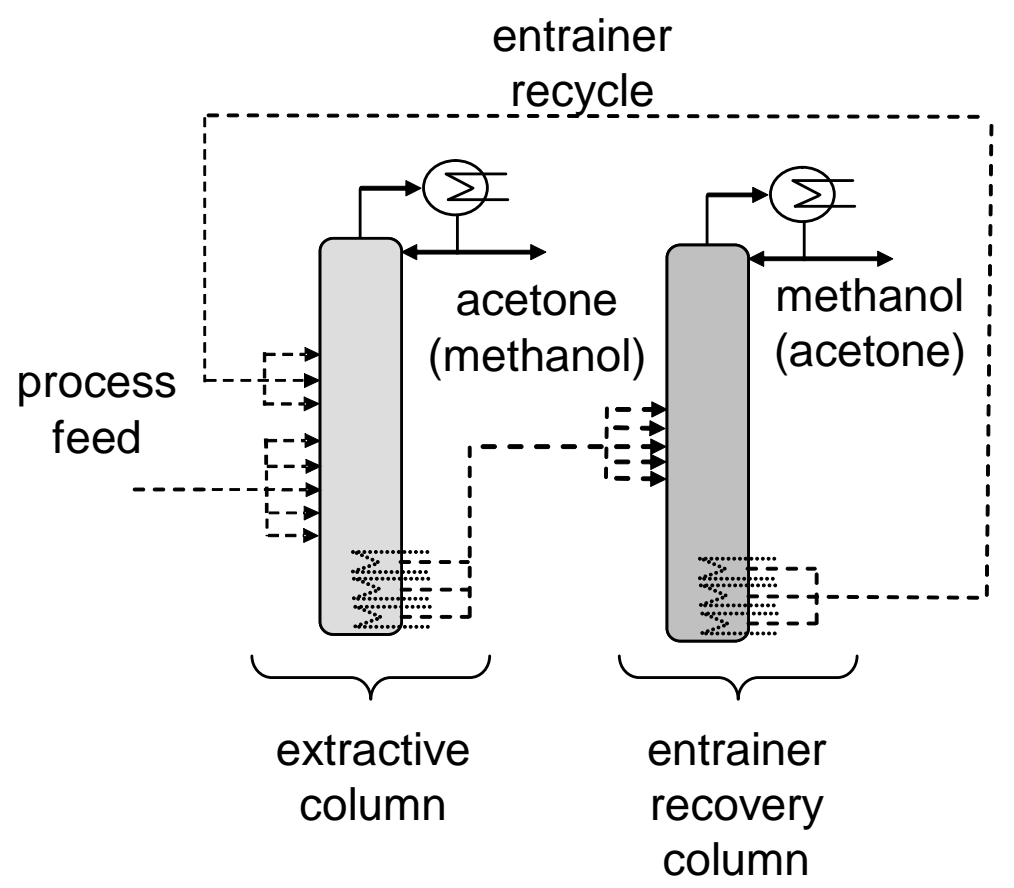

Figure 1: Sketch of an extractive distillation setup. The process feed is separated with the help of an entrainer in the extractive distillation column, yielding one azeotrope forming component as the distillate. In the second column the other component is separated from the entrainer.

In classical extractive distillation setup (cf. Fig. 1), one azeotrope forming component is withdrawn at the top of the extractive column, while the other, together with the entrainer, forms the bottoms product. In a second column, the entrainer is separated from the second component and recycled to the first column. The separation in the second column is easier when a large difference in the boiling points between the high-boiling entrainer and the second component exists and no additional azeotropes occur in the mixture.

Extractive distillation can be more energy efficient than azeotropic separation, where a liquid phase split is used to overcome distillation boundaries (18). This is especially true when thermally integrated sequences are used (12). Using extractive distillation, however, high product purities are sometimes difficult to obtain (18). This is apparently the reason why about $45 \%$ of all industrial distillation assignments are either conventional or azeotropic setups while only $10 \%$ are operated as extractive distillation (3). 
Another drawback of extractive distillation is the larger number of degrees of freedom when compared with a simple distillation setup. In a simple distillation setup these degrees of freedom are the reflux ratios and the number of stages of the distillation columns. For extractive distillation the entrainer choice and the entrainer flowrate comprise additional degrees of freedom.

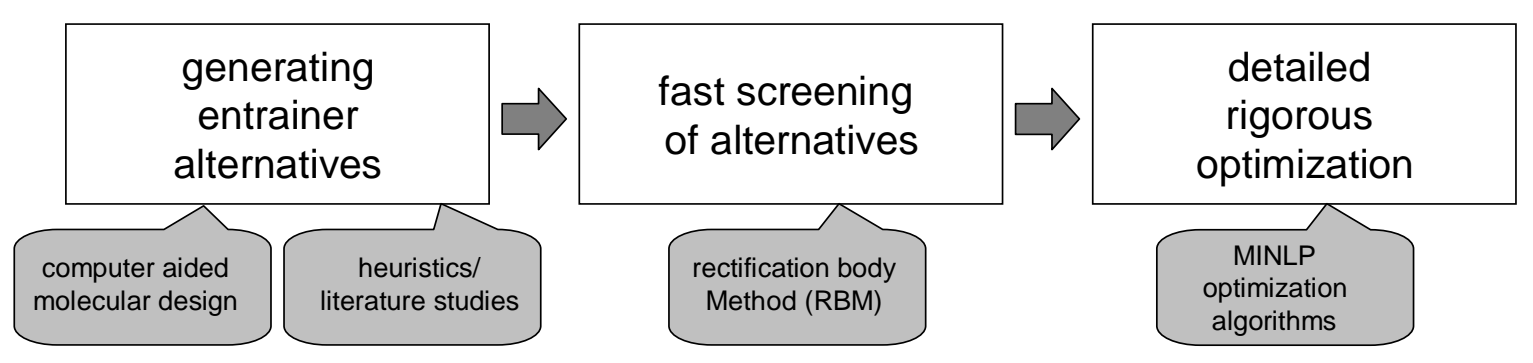

Figure 2: Outline of the proposed synthesis framework

To facilitate an easier conceptual design of distillation processes a systematic synthesis framework has been proposed (13). This framework, which has so far only been applied to simple distillation, is extended here to extractive distillation. As outlined in Fig. 2, the entrainer candidates can either be generated systematically through Computer Aided Molecular Design (CAMD) or found from heuristic rules or literature studies. The resulting flowsheets for the different entrainer candidates are screened next to find the economically most attractive entrainers. Different screening methods are reviewed; it is shown that shortcut calculations with an extended RBM (4) give the most meaningful screening results. Furthermore, the results from this shortcut step can be used to initialize a more detailed MINLP optimization of the entire extractive flowsheet (14). This MINLP optimization marks the concluding step of the systematic synthesis framework. After completion of these steps the optimal entrainer choice, the optimal column design and the optimal operating point have been found.

The methods presented throughout the paper are illustrated with the sample separation of an acetone/methanol mixture. This mixture is typically separated through extractive distillation using water as an entrainer (5) to produce acetone as the distillate of the extractive column (cf. Fig. 1). The feed concentration of the example process is assumed to be $0.7774 / 0.2226$ and the feed flowrate is assumed to be $1 \mathrm{kmol} / \mathrm{s}$. The vapor phase was modeled as an ideal gas. For the evaluation of the minimum energy demand in Section 4.2.2, all feeds to the individual columns were assumed to be boiling liquids.

In the next section, a brief summary of the thermodynamic foundations of extractive distillation is given. Methods to find possible entrainer candidates are presented in Section 3. Section 4 focuses on methods for the rapid screening and evaluation of entrainer candidates, while the MINLP optimization strategy based on rigorous models is presented in Section 5 . 


\section{Extractive distillation with liquid entrainers}

\subsection{Thermodynamic foundations}

The relative volatility of the key components $i$ and $j$ in a given mixture with ideal vapor phase is defined by

$$
\alpha_{i j}=\frac{y_{i} / x_{i}}{y_{j} / x_{j}}=\frac{\gamma_{i} p_{i}^{0}}{\gamma_{j} p_{j}^{0}},
$$

where $x$ and $y$ are the molar fractions in the liquid and vapor phase, $\gamma$ is the activity coefficient and $p^{0}$ the pure component vapor pressure.

Since the ratio $p_{i}^{0} / p_{j}^{0}$ is constant for a given temperature, the solvent only affects the ratio of the activity coefficients $\gamma_{i} / \gamma_{j}$. In the presence of a solvent $S$, this ratio is called selectivity $S_{i j}(3)$ :

$$
S_{i j}=\left(\frac{\gamma_{i}}{\gamma_{j}}\right)_{S}
$$

The activity coefficients depend on the liquid phase composition. Since the effect of the entrainer tends to increase with concentration in the mixture, it is common practice to evaluate the selectivity at infinite dilution (18). The definition of selectivity given in eq. (2) then becomes

$$
S_{i j}^{\infty}=\left(\frac{\gamma_{i}^{\infty}}{\gamma_{j}^{\infty}}\right),
$$

which also represents the maximum possible selectivity ${ }^{2}$.

Another proposed measure to asses the suitability of an entrainer is the capacity (9) which is determined by

$$
C_{j, \text { Entrainer }}^{\infty}=\frac{1}{\gamma_{j}^{\infty}},
$$

where $j$ denotes the solute. The smaller the value of the activity coefficient $\gamma_{j}^{\infty}$, the stronger are the interactions between component $j$ and the entrainer, which results in a larger capacity $C_{j, \text { Entrainer }}^{\infty}$. Jork et al. (9) note that entrainers with a high selectivity often possess a low capacity. Mostly, however, the capacity is neglected (cf. $(3 ; 18)$ ). We follow this view and see the capacity as a secondary decision variable; it is therefore not included in our screening process. We will, however, review the different screening criteria at the end of this paper.

While a high selectivity is certainly the most important criterion for the selection of an entrainer, several other constraints also need to be fulfilled:

1. The entrainer should not form any new azeotropes with the original components (3).

2. The entrainer should not cause any immiscible regions (3).

3. The boiling point should differ significantly from the other components to facilitate an easy separation in the second column (3).

\footnotetext{
${ }^{2}$ For the evaluation of eq. (3) we set $x_{i}=x_{j}=0(i \neq j)$ and $x_{S}=1$, where $S$ denotes the solvent in excess.
} 
4. Entrainer selection should also consider safety, environmental effects, corrosion, price and availability (18).

There are classes of entrainers that facilitate the separation even though new azeotropes are formed and criterion 1 is violated (16). The analysis presented here is restricted to the class of heavy boiling entrainers that do not form any new azeotropes, since this is the most relevant setting for extractive distillation $(5 ; 16 ; 17 ; 18)$.

\subsection{Nonlinear analysis of extractive distillation}

A certain minimum entrainer flow rate $E_{\min }$ is required to make an extractive separation feasible. This minimum entrainer flow can be calculated from a bifurcation analysis of the nonlinear tray-to-tray (11) or pinch equations (4). It is also known that not only a minimum reflux ratio $r_{\min }$ is required, but also a maximum reflux ratio $r_{\max }$ exists above which the desired separation is infeasible. These values can be determined from the rectification body method for extractive separation (see Section 4.2.2 and (4)).

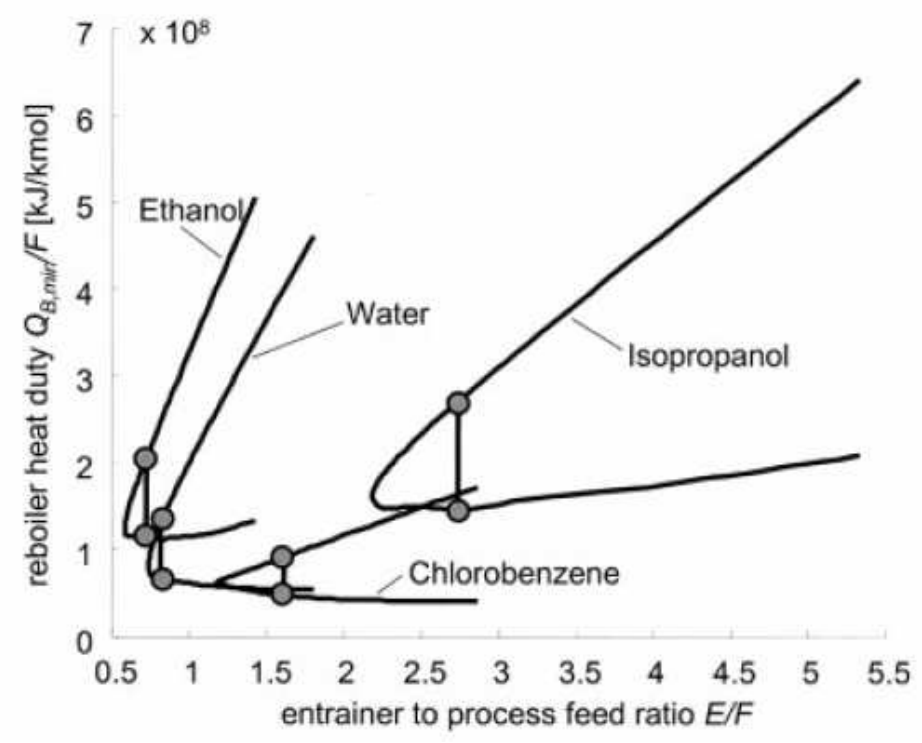

Figure 3: Minimum and maximum reflux ratio as a function of entrainer to process feed ratio. The dots denote $Q_{B, \min }$ and $Q_{B, \max }$ at $E / E_{\text {min }} \geq 1.1$ and $r_{\max } / r_{\min } \geq 2.0$. Figure taken from (4).

When the minimum and maximum reboiler heat duties (corresponding to the minimum and maximum reflux) for the sample separation of acetone and methanol are plotted for different entrainer candidates as a function of the entrainer flowrate (cf. Fig. 3), the minimum reflux ratio is usually minimal at the minimum entrainer flowrate. One notable exception is chlorobenzene, where the minimum reflux ratio and therefore the minimum energy demand is significantly lower at higher entrainer flowrates (cf. fig. 3). In the extractive column, substantial energy savings would be possible if a higher entrainer flow is chosen. This increased entrainer flow rate would lead to a higher energy demand in the entrainer recovery column. In case of chlorobenzene, however, the decrease in energy demand in the extractive column 
offsets the increase in energy demand of the entrainer recovery column. This behavior has also been found for p-Xylene, when the mixture is modeled using the UNIFAC parameters. Although this potential for energy saving is not very common, it is recommended to perform a search with increasing entrainer flow rate to find the optimal value (cf. 4.1.3). This enables the comparison of different flowsheets at their optimal operating point and provides even better initial values for the MINLP optimization (cf. Section 5).

\section{Entrainer selection}

This section focuses on methods to find promising entrainer candidates. We restrict ourselves to the industrially most relevant case of organic liquid entrainers and water for the time being.

\subsection{Heuristics}

The extractive property of an entrainer is based on the ability to form hydrogen bonds with one of the key components (18). Based on this property, entrainer candidates can be classified into five groups. The classification is derived from the enthalpy of vaporization of a component in comparison with Trouton's constant $\left(\Delta H_{\text {vap }}=88 \mathrm{~J} / \mathrm{K} \cdot \mathrm{mol}\right)$ : a larger value indicates a large interaction in the liquid phase while a lower value usually indicates the formation of associates in the vapor phase. Blass (3) introduces the following different classes:

- Class I includes molecules which are capable to form three-dimensional networks of strong hydrogen bonds in the liquid phase. They show the highest deviation from Trouton's constant. Components such as water $\left(\Delta H_{v a p}=109 \mathrm{~J} / \mathrm{K} \cdot \mathrm{mol}\right)$, gly$\operatorname{col}\left(\Delta H_{\text {vap }}=121,1 \mathrm{~J} / \mathrm{K} \cdot \mathrm{mol}\right)$, hydroxylamine, hydroxyaxcids, amides etc. belong to this group.

- Class II includes molecules which are capable to form one-dimensional chains through hydrogen bonds. For these components, the effect of hydrogen bonds is not as predominant as for those in class I and van der Waals' forces become more important. Components such as ethanol $\left(\Delta H_{\text {vap }}=110,1 \mathrm{~J} / \mathrm{K} \cdot \mathrm{mol}\right)$ and acetic acid $\left(\Delta H_{\text {vap }}=60,5 \mathrm{~J} / \mathrm{K} \cdot \mathrm{mol}\right)$ belong to this group.

- Class III includes molecules that act as a hydrogen bond acceptor. Ethers, ketones and nucleophilic aromatic compounds are included in this group. $\Delta H_{v a p}$ is close to Trouton's constant for molecules belonging to this group.

- Class IV consists of molecules that act primarily as hydrogen bond donors, such as chloroform, dichlorethan, trichlorethan etc.

- Molecules in Class $V$ do neither act as hydrogen bond donors nor as acceptors. Tetrachlorethan and aromatic compounds belong to this group.

Table 1 shows the deviations from Raoult's law for the different interacting component groups. The best entrainers should be found in component classes I \& II (18), which cause a positive deviation form Raoult's law. It should be noted that there are many exceptions to 
these heuristics. Special care needs to be taken to avoid the formation of new azeotropes and heterogeneous regions, as can be seen in Table 1 . This is usually tested through the measurement of VLE data or through gas-liquid chromatography (18). These two procedures are, however, time consuming.

\begin{tabular}{|c|c|c|}
\hline $\begin{array}{c}\text { interacting } \\
\text { Classes }\end{array}$ & Deviation & H-Bonding \\
\hline $\begin{array}{l}\mathrm{I}+\mathrm{V} \\
\mathrm{II}+\mathrm{V}\end{array}$ & $\begin{array}{l}\text { Always positive; } \mathrm{I}+\mathrm{V} \text { usually } \\
\text { show phase splitting }\end{array}$ & $\begin{array}{l}\text { H-Bonds are } \\
\text { dissociated }\end{array}$ \\
\hline $\mathrm{III}+\mathrm{IV}$ & Always negative & H-Bonds are formed \\
\hline $\begin{array}{l}\text { I + IV } \\
\text { II + IV }\end{array}$ & $\begin{array}{l}\text { Always positive; I + IV usually } \\
\text { show phase splitting }\end{array}$ & $\begin{array}{c}\text { More H-Bonds are } \\
\text { dissociated than formed }\end{array}$ \\
\hline $\begin{array}{c}\mathrm{I}+\mathrm{I} \\
\mathrm{I}+\mathrm{II} \\
\mathrm{I}+\mathrm{III} \\
\\
\mathrm{II}+\mathrm{II} \\
\mathrm{II}+\mathrm{III}\end{array}$ & $\begin{array}{l}\text { Usually positive, } \\
\text { sometimes negative } \\
\text { and formation of } \\
\text { maximum azeotropes }\end{array}$ & $\begin{array}{l}\text { Some H-Bonds } \\
\text { are formed, } \\
\text { some areS } \\
\text { dissociated }\end{array}$ \\
\hline $\begin{array}{c}\text { III + III } \\
\text { III + V } \\
\text { IV + IV } \\
\text { IV + V } \\
V+V\end{array}$ & $\begin{array}{c}\text { Nearly ideal } \\
\text { systems; small positive } \\
\text { deviations, seldom } \\
\text { formation of minimum } \\
\text { azeotropes }\end{array}$ & $\begin{array}{l}\text { No Hydrogen } \\
\text { bonds }\end{array}$ \\
\hline
\end{tabular}

Table 1: Deviation from Raoult's law for different classes of interacting components (taken from (3))

\subsection{Computer Aided Molecular Design}

While heuristic guidelines offer a way to find entrainer candidates, they are usually not very efficient. Computer aided molecular design (CAMD) on the other hand is a systematic tool for efficient design of entrainer candidates (10). CAMD can be interpreted as the inverse of the property prediction problem. Given a set of desirable properties - in this case a high selectivity for one component - a combination of structural groups is sought that satisfies the property specification. The application of CAMD for entrainer design is mostly based on the UNIFAC group contribution method. Two different approaches to molecular design, namely optimization-based and generate-and-test approaches, can be distinguished (6). Both alternatives are briefly outlined in the following.

\subsubsection{Optimization-based CAMD}

The entrainer design problem can be formulated as a MINLP problem, where the selectivity is maximized subject to several constraints (10). The most important constraints in the context of entrainer design are structural constraints, pure component property constraints and mixture property constraints. 
Structural constraints are rules for the generation of physically meaningful components from a collection of groups. These constraints typically include (10)

- the octet rule, which ensures that the product has zero valency,

- structural feasibility constraints that ensure that two adjacent groups in a molecule are not linked by more than one bond, and

- constraints on lower and upper limits of the number of groups of a particular type and on the total number of groups in a molecule.

Pure component property constraints usually include the boiling point difference, while mixture property constraints aim to avoid liquid-liquid phase splitting or the formation of new azeotropes. For the evaluation of these properties, group contribution methods such as Joback or UNIFAC are typically employed. The high complexity of the optimization problem has led to the development of decomposition methods for optimization based CAMD (10). Here, subproblems with increasing complexity are solved to provide a numerically stable CAMD method.

\subsubsection{CAMD by generate-and-test}

Another approach to computer aided molecular design is called generate-and-test. It consists of three basic steps $(7 ; 8)$ :

1. Pre-Design: Define the problem in terms of desired properties of the compound to be designed.

2. Design: Run the actual CAMD design algorithm to generate compounds and test them against stated criteria from the pre-design stage.

3. Post-Design: Test the results based on properties that are not easily screened during stage two, such as environmental and safety criteria.

Each of these three steps will be described briefly. For a more detailed discussion, the reader is referred to Harper and Gani $(7 ; 8)$.

\section{Pre-Design}

In this step, the desired and the undesired properties of the new molecules are specified as targets for the generation algorithm. In case of entrainer design, the target value is a high selectivity at infinite dilution as defined in eq. (3). Further target properties are a high boiling point, no miscibility gap with the components in the original mixture and no formation of new azeotropes (cf. Section 2.1). All these constraints can be specified in ICAS (7), a software package that can readily be used for CAMD applications. 
Design

Given the targets from the pre-design phase, feasible molecules are generated. Again a set of rules as outlined in Section 3.2.1 is required to design only meaningful compounds. The algorithm used here splits the problem into a number of sub-problems with increasing complexity (7). The generated molecules can then be ordered by a variety of criteria, for example by the selectivity at infinite dilution $S_{i j}^{\infty}$ or by more sophisticated functions to prepare the post-design phase.

Post-Design

In the post-design phase the user can rank and test the generated alternatives. In Section 4 different ranking and test criteria will be presented and compared.

\subsection{Entrainer candidates for the illustrative case study}

\subsubsection{Literature study}

For the sample separation of acetone and methanol several entrainer candidates can be found in the literature. Laroche et al. (16) suggest water, ethanol, isopropanol and chlorobenzene, while Lei et al. (18) additionally recommend ethylene glycol. Employing the heuristics reviewed in Section 3.1, it can be expected that the polar components associate with methanol and produce acetone as the distillate product in the extractive column. The unpolar chlorobenzene, on the other hand, binds with acetone and produces high purity methanol as the distillate product in the extractive column, even though methanol is the intermediateboiling component in the original mixture.

\subsection{2. $C A M D$}

The following specifications are set in the ICAS module ProCAMD which uses a generate and test approach (cf. Section 3.2.2) to generate molecules:

- For methanol as the solute (and acetone as the distillate product of the extractive column), only acyclic alkanes and alcohols with a maximum number of eight functional groups should be designed.

- For acetone as the solute (and methanol as the distillate product of the extractive column), only acyclic alkanes and compounds that contain chlorine with a maximum number of eight functional groups should be designed.

These compound design specifications stem from an evaluation of the heuristic guidelines presented in Section 3.1. These two constraints decrease the search space, but, as shown below, still a large number of feasible and useful entrainer candidates are designed in both cases. Other specifications include:

- The minimum boiling temperature for the entrainer candidates is set to $350 \mathrm{~K}$ to facilitate an easy separation in the entrainer recovery column. Furthermore, a maximum boiling temperature of $500 \mathrm{~K}$ is specified. 
- As stated in Section 2.1, no miscibility gap and no additional azeotropes should be introduced by the entrainer. The criterion for phase splitting is set so that an entrainer flowrate of $1.4 \mathrm{E} / \mathrm{F}$ does not cause any immiscibility with the feed. It should be noted that this miscibility check can also be done for a range of compositions and temperatures.

- A minimum selectivity of $S_{i j}^{\infty}>2.5$ as predicted with the UNIFAC model is requested in both cases.

- As suggested in Section 3.2.2, the generated candidates are checked if they have been included in the DIPPR (20) or ASPEN (1) databases ${ }^{3}$. If they could not be found in the databases, they were excluded pragmatically from further considerations.

Common solvents are screened in addition to the generation of new components with ProCAMD. The results are given in Tables 2 and 3.

\begin{tabular}{|l|c|c|c|}
\hline \multicolumn{4}{|c|}{ distillate acetone } \\
\hline $\begin{array}{l}\text { entrainer } \\
\text { candidate }\end{array}$ & $\begin{array}{c}S_{i j}^{\infty}[-] \\
\text { UNIFAC }\end{array}$ & $\begin{array}{c}S_{i j}^{\infty}[-] \\
\text { UNIQUAC }\end{array}$ & $\begin{array}{c}T_{\text {boil }}[\mathrm{K}] \\
(20)\end{array}$ \\
\hline water & 4.81 & 2.42 & 373.15 \\
\hline DMSO & 1.47 & 2.89 & 464.0 \\
\hline ethylene glycol & 2.99 & 4.19 & 470.45 \\
\hline ethanol & 1.76 & 1.65 & 351.44 \\
\hline isopropanol & 1.3 & 1.72 & 355.41 \\
\hline
\end{tabular}

Table 2: Entrainer candidates generated for the acteone/methanol separation; listed are entrainers which bond with methanol and therefore produce acetone as the distillate.

As shown in Table 2, the entrainer candidates reported in the literature (cf. Section 3.3.1.) for methanol could be reproduced with the CAMD algorithm. The notable exception is ethanol, which is apparently excluded because the boiling point estimate is $330.1 \mathrm{~K}$ (which is less than the required $350 \mathrm{~K}$ ), even though the measured boiling point of ethanol is above that threshold. For known solvents the screening should therefore be based on experimental data. DMSO (dimethylsulphuroxide), ethanol and isopropanol were also excluded from the list of possible entrainer candidates because their selectivity is below the required value of 2.5 according to the UNIFAC model. They are left in the sample, however, to retain a larger number of entrainer candidates to test the entrainer selection criteria in the next section.

When the criterion for the minimum required selectivity is relaxed to 2.0 , a number of diols (propane-, butane- and pentanediol) and n-methyl-2-pyrrolidone are designed. However, we have decided to keep the bound on $S_{i j}^{\infty}$ the same as in the case where acetone is the distillate of the extractive column (see next section).

\footnotetext{
${ }^{3}$ For a continuous chemical process usually a large amount of entrainer material is required. Availability may be a problem for some of the more exotic chemical compounds designed in the CAMD step. Selecting components from these databases also has the additional advantage that constants for pure component vapor pressure correlations are available, which increases the reliability of the predictions tremendously. This criterion could, however, lead to the exclusion of very promising yet uncommon compounds.
} 


\begin{tabular}{|l|c|c|c|}
\hline \multicolumn{4}{|c|}{ distillate methanol } \\
\hline $\begin{array}{l}\text { entrainer } \\
\text { candidate }\end{array}$ & $\begin{array}{c}S_{i j}^{\infty}[-] \\
\text { UNIFAC }\end{array}$ & $\begin{array}{c}S_{i j}^{\infty}[-] \\
\text { UNIQUAC }\end{array}$ & $\begin{array}{c}T_{\text {boil }}[\mathrm{K}] \\
((20))\end{array}$ \\
\hline ethylbenzene & 4.59 & 1.04 & 409.35 \\
\hline m-xylene & 4.4 & NA & 412.27 \\
\hline o-xylene & 4.4 & NA & 417.58 \\
\hline p-xylene & 4.4 & 2.32 & 411.51 \\
\hline mesitylene & 3.75 & NA & 437.89 \\
\hline 1,2,3-trimethylbenzene & 3.59 & NA & 449.27 \\
\hline $1,2,4$-trimethylbenzene & 3.59 & NA & 442.53 \\
\hline benzyl ethyl ether & 2.53 & NA & 458.15 \\
\hline \hline chlorobenzene & 5.51 & 5.84 & 404.87 \\
\hline
\end{tabular}

Table 3: Entrainer candidates generated for the acteone/methanol separation; listed are entrainers which bond with acetone and therefore produce methanol as the distillate. Chlorobenzene is listed under the double line since it was manually re-included in the sample (see text).

When methanol is the desired distillate product, the entrainer candidates given in Table 3 are generated. Chlorobenzene, which has been previously suggested by Laroche et al. (16) (cf. Section 3.3.1.), is excluded by the ProCAMD algorithm because the UNIFAC parameters wrongly predict the formation of an azeotrope. Since it has a very high predicted selectivity of 5.51, it is retained in the sample.

The selectivities at infinite dilution of some candidates in Table 3 are notably higher than those given in Table 2. The bound of $S_{i j}^{\infty}>2.5$ is not as strict in this case and more entrainer candidates would be expected. However, only a few physically meaningful structures are generated. For those found, often ASPEN parameters are not available which led to the exclusion of dibromoethane, dichlorobutane and dichloropentane.

\section{Entrainer screening}

From the several entrainer alternatives the economically best alternative giving the lowest total annualized cost (TAC) of the process has to be determined. This requires, however, a detailed MINLP optimization of the entire extractive distillation process as done by Kraemer et. al. (15), which is not practical for a large number of entrainer alternatives. It is therefore advisable to screen the entrainer alternatives based on shortcut calculations to narrow the choices down to two or three promising alternatives first. For such a small number of possible entrainers, a complete rigorous optimization and costing can be done to reliably identify the best entrainer. For the screening of entrainer alternatives a number of criteria, either based on thermodynamics or shortcut calculations have been devised. In the following these screening methods are applied to the sample separation. The main ideas, requirements and results are briefly outlined for each method. For a more detailed discussion the reader is referred to the cited literature. 


\subsection{Screening methods}

\subsubsection{Thermodynamic considerations}

As stated in Section 2.1, the selectivity at infinite dilution $S_{i j}^{\infty}$ is a very important selection criterion for different entrainer candidates, even though finite dilution is the prevailing condition in actual columns. Laroche et al. (16) note that the selectivity at finite dilution and the selectivity at infinite dilution are not necessarily related to each other. Some entrainers may even exhibit a maximum selectivity at an intermediate concentration (16). Nevertheless, the entrainer selection based on selectivity at infinite dilution is still the most commonly applied criterion.

The influence of the entrainer selectivity on the total annualized cost of the process has been studied in great detail by Momoh (19). The mixtures of n-butane/trans-2-butene and n-hexane/benzene were used as sample problems. For each mixture and choice of entrainer a complete design and costing was done. Process optimization was done manually through iterative simulation studies. Some results of Momoh (19) are presented in Fig. 4, where it can be seen that the TAC decreases with increasing entrainer selectivity in general. However, significant discrepancies from this trend can be observed in both cases. It can be concluded from this study that the entrainer with the highest selectivity has a good potential to be the best choice for the overall process, while some other factors such as the heat of vaporization of the entrainer may also constitute an important contribution to the total process cost.

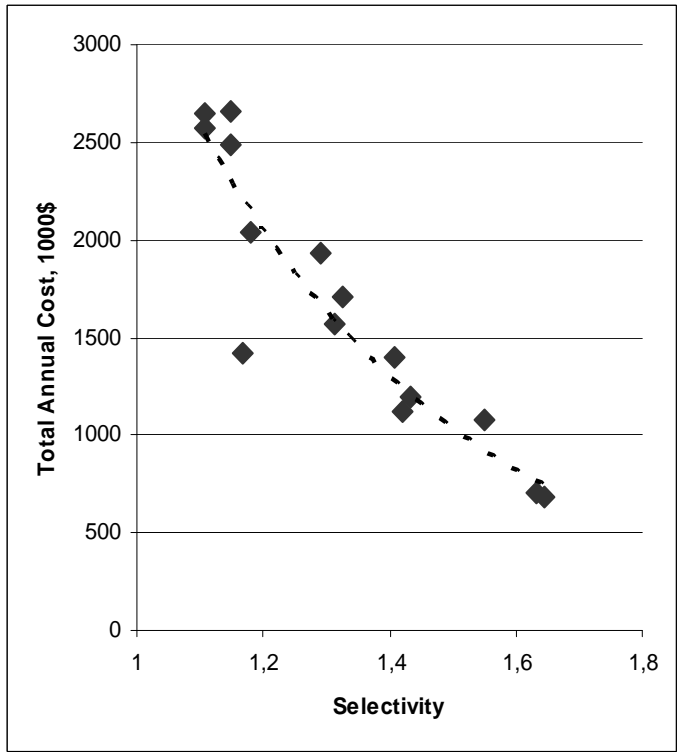

(a)

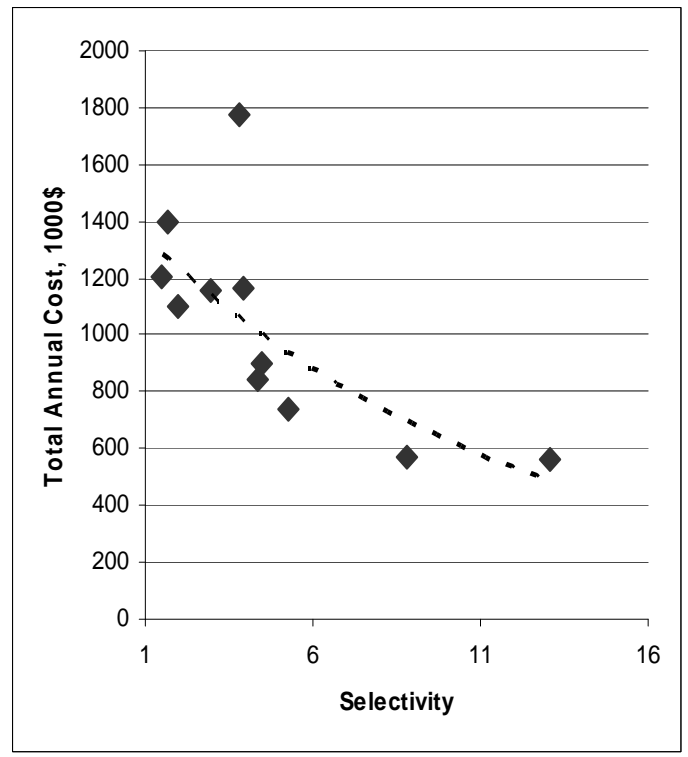

(b)

Figure 4: The effect of entrainer selectivity on the TAC of an extractive distillation process. (a) n-butane/trans-2-butene mixture and (b) n-hexane/benzene mixture; adapted from Momoh (19)

This extremely simple method allows a fast screening of a large number of different entrainer alternatives. For a given separation and for a fixed flowsheet topology this crite- 
rion might be sufficient. When, as in the sample separation of acetone and methanol the distillate product of the extractive column changes with the choice of entrainer, no direct comparison is possible. As will be shown below, the selectivity is only suited for a comparison of structurally identical flowsheets. When the distillate product changes from one entrainer alternative to another, a meaningful ranking is not possible. Momoh (19) also notes that choosing solvents on the basis of selectivity alone tends to emphasize the cost of the extractive column, whereas the cost of the second column is neglected. This cost can, however, dominate the total process cost in some cases.

This method is therefore very well suited as an objective function in CAMD or for a prescreening of a large number of different entrainer alternatives, while the results are not as detailed and meaningful as those obtained with the more advanced methods. For example, screening with this simple criterion does not give an indication of the minimum entrainer flowrate or the minimum and maximum reflux (cf. Section 2.2).

\subsubsection{The Rectification Body Method for extractive columns}

The Rectification Body Method (RBM) (2) allows the computation of the minimum separation energy of azeotropic mixtures with an arbitrary number of components. This method is based on approximating column profiles through the so-called rectification bodies, which can be calculated from pinch points. Recently, this tool has been extended to extractive distillation processes (4).

This method is divided into two distinct steps. In the first step, the minimum entrainer flowrate is determined from the analysis of the nonlinear pinch equations. It could be shown that a so-called pitchfork bifurcation appears at a critical reflux ratio and at the minimum entrainer flowrate. This minimum entrainer flowrate is called $E_{\text {min }, b i f}$. At this entrainer flowrate the ratio of the feasible reflux ratios $r_{\min }$ and $r_{\max }$ is usually very small, making the process extremely sensitive to small changes in the feed. Brüggemann and Marquardt (4) therefore propose operational constraints of $r_{\max } / r_{\min }=2.0$ and $E / E_{\min , b i f}=1.1$ to guarantee stable process operation. Here $r_{\max }$ is determined from the nonlinear analysis and $r_{\text {min }}$ from a tailored RBM. The entrainer flowrate that obeys these additional constraints will be denoted by $E_{\min }$.

The main advantage of this method computed to the thermodynamical criteria presented in the previous section is its capability to predict the minimum energy demand of the separation. This allows a direct comparison of extractive distillation to other flowsheet alternatives. The method can also determine the minimum flow rate of the entrainer from a nonlinear analysis of the pinch equations (4). The RBM gives the most accurate information of all the screening methods presented. As shown by Brüggemann and Marquardt (4), the RBM results are very well reproducible with the flowsheet simulator package ASPEN.

\subsubsection{Flowsheet optimization using shortcut models}

As noted in Section 2.2, an increase in entrainer flowrate might yield a lower energy demand of the entire process. To compare the different entrainer alternatives at the optimal operating point, a simple flowsheet optimization is run after the determination of the energy demand of the individual columns. 
Here, the only optimization variable is the entrainer flowrate, since the overall mass balance and the compositions of the individual product streams are fixed. Therefore a simple one dimensional search along increasing entrainer flowrates is sufficient in this case. As could already be deduced from Fig. 3, the only case in which a substantial energy reduction could be observed is when chlorobenzene and p-xylene (when the UNIFAC model is used) are selected as an entrainer. Since the computational cost of this step is very low it is advised to run this optimization step anyway to at least improve the initialization values for the following MINLP optimization step (cf. Section 5.2).

It should be noted here that this step is very important when a more complex flowsheet with a mixed recycle stream is designed. As shown previously (13) this step can lead to substantial savings in energy and reduces the computational time of the MINLP optimization significantly.

\subsection{Evaluating entrainer candidates for the case study}

\subsubsection{Ranking by selectivity}

The selectivities at infinite dilution, $S_{i j}^{\infty}$, as calculated with the UNIFAC model and, where parameters were available in ASPEN, with the UNIQUAC model, are also listed in Tables 2 and 3 for the different entrainer candidates.

Two interesting conclusions can be drawn from these simple calculations:

1. The calculated selectivities as predicted with UNIFAC and those calculated with the UNIQUAC model seem to be in reasonably good agreement. In case of ethylbenzene, and to a lesser extent in case of ethylene glycol, a large discrepancy is observed. A satisfying reason for this behavior could not be found.

2. The numerical value of $S_{i j}^{\infty}$ is much smaller for all those candidates that bind with methanol than for those that bind with acetone. This should be the reason why a smaller number of entrainer candidates could be found in this case.

If the final entrainer candidate was chosen based on the evaluation of the selectivity at infinite dilution, the best entrainer alternatives would be any candidate found in Table 3 . As mentioned above, all entrainer candidates listed in Table 2 seem to be inferior and should be neglected. In industrial practice, however, the only entrainer candidates found are those listed in Table 2 that produce acetone as the distillate in the extractive section. One reason is that water and ethyl glycol are environmentally benign substances and are therefore preferred. Another reason can be deduced from the more thorough analysis presented in the next sections.

\subsubsection{Ranking by the RBM}

The RBM for extractive distillation is used as a second screening criterion for the sample separation. Only one of the xylene isomers (p-xylene) and one of the trimethylbenzenes isomers (1,2,3-trimethylbenzene) is chosen since all isomers are described by the same UNIFAC groups. The small differences in the pure component vapor pressures between the different isomers are neglected. Since UNIQUAC is used whenever possible, ethylbenzene is also excluded from the list, since it does not fulfill the criterion $S_{i j}^{\infty}>2.5$. 


\begin{tabular}{|l|c|c|c|c|c|}
\hline \multicolumn{7}{|c|}{ distillate acetone } \\
\hline $\begin{array}{l}\text { entrainer } \\
\text { candidate }\end{array}$ & $S_{i j}^{\infty}$ & $E_{\text {min,bif }} / F$ & $E / F$ & $\begin{array}{c}Q_{B, \min } / F \\
\text { extractive column }\end{array}$ & $\begin{array}{c}Q_{B, \min } / F \\
\text { total process }\end{array}$ \\
\hline DMSO & 2.89 & 0.651 & 0.771 & 5.42 & 7.20 \\
\hline water & 2.4 & 0.814 & 0.933 & 9.866 & 11.803 \\
\hline ethanol & 1.65 & 0.721 & 0.760 & 11.077 & 16.981 \\
\hline isopropanol & 1.71 & 2.187 & 2.923 & 15.97 & 26.101 \\
\hline \hline ethylene glycol & 4.19 & NA & NA & NA & NA \\
\hline \hline \multicolumn{7}{|c|}{ distillate methanol } \\
\hline chlorobenzene & 5.84 & 1.615 & 2.015 & 3.75 & 7.93 \\
\hline p-xylene (UNIFAC) & 4.4 & 0.81 & 1.67 & 3.82 & 8.71 \\
\hline mesitylene & 3.75 & 0.82 & 1.55 & 4.01 & 9.562 \\
\hline p-xylene (UNIQUAC) & 2.32 & 0.77 & 2.38 & 4.32 & 9.94 \\
\hline $1,2,3$-trimethylbenzene & 3.59 & 0.83 & 1.63 & 3.93 & 10.11 \\
\hline \hline benzyl ethyl ether & 2.53 & 1.13 & NA & NA & NA \\
\hline
\end{tabular}

Table 4: Results for the different entrainer alternatives obtained with the RBM for extractive columns after the flowsheet optimization. The total process also takes the energy demand of the entrainer recovery column into consideration. For the components below the double line RBM for extractive distillation did not find a minimum entrainer flowrate (ethylene glycol) or reflux bounds (benzyl ethyl ether). All energies are given in $10^{7} \mathrm{~W} / \mathrm{kmol}$.

The results calculated with the RBM are given in Table $4^{4}$. The nonlinear analysis of the pinch equations does not yield a minimum entrainer flowrate for ethylene glycol. Furthermore, no reflux bounds can be found for benzyl ethyl ether.

For all other entrainer alternatives the minimum flowrate $E_{\text {min,bif }}$ as well as the minimum entrainer flowrate $E$ that satisfies the constraints stated in Section 4.1.2 are given. Furthermore, the minimum reboiler energy $Q_{B, \min } / F$ for the extractive column and the minimum energy demand of the total process, which also includes the entrainer recovery column, after the flowsheet optimization are listed.

As mentioned in Section 4.1.3. the minimum energy demand of the process can be decreased in rare cases when the entrainer flowrate is increased. An optimization along increasing entrainer flowrates yields that this is the case for chlorobenzene and p-xylene UNIFAC-model. In case of chlorobenzene for example an increase in entrainer flowrate from $E_{\min }=1.615$ to 2.015 decreases the energy demand of the entire process from 8.079. $10^{7} \mathrm{~W} / \mathrm{kmol}$ to $7.934 \cdot 10^{7}$. For all subsequent consideration this lower energy demand is used.

As can be seen in Table 4, the comparison of different entrainers based on their selectivity allows only an approximate ranking. As the process parameters change, i.e. a different distillate product is chosen, no conclusions can be drawn from this parameter alone. Based on selectivity alone, isopropanol should be a better entrainer than ethanol. The results obtained with the RBM, however, show that the opposite is true.

\footnotetext{
${ }^{4}$ The values differ from those given in (4) due to a slightly improved algorithm and, in some cases, e.g. water, because of different physical property parameters.
} 
Another interesting observation stems from a comparison p-xylene (UNIFAC) and pxylene (UNIQUAC). While the total process energy demands are not very different, the sensitivities show a large difference. While UNIQUAC parameters should be preferred, a first screening can be done reliably with the UNIFAC model at least in this case.

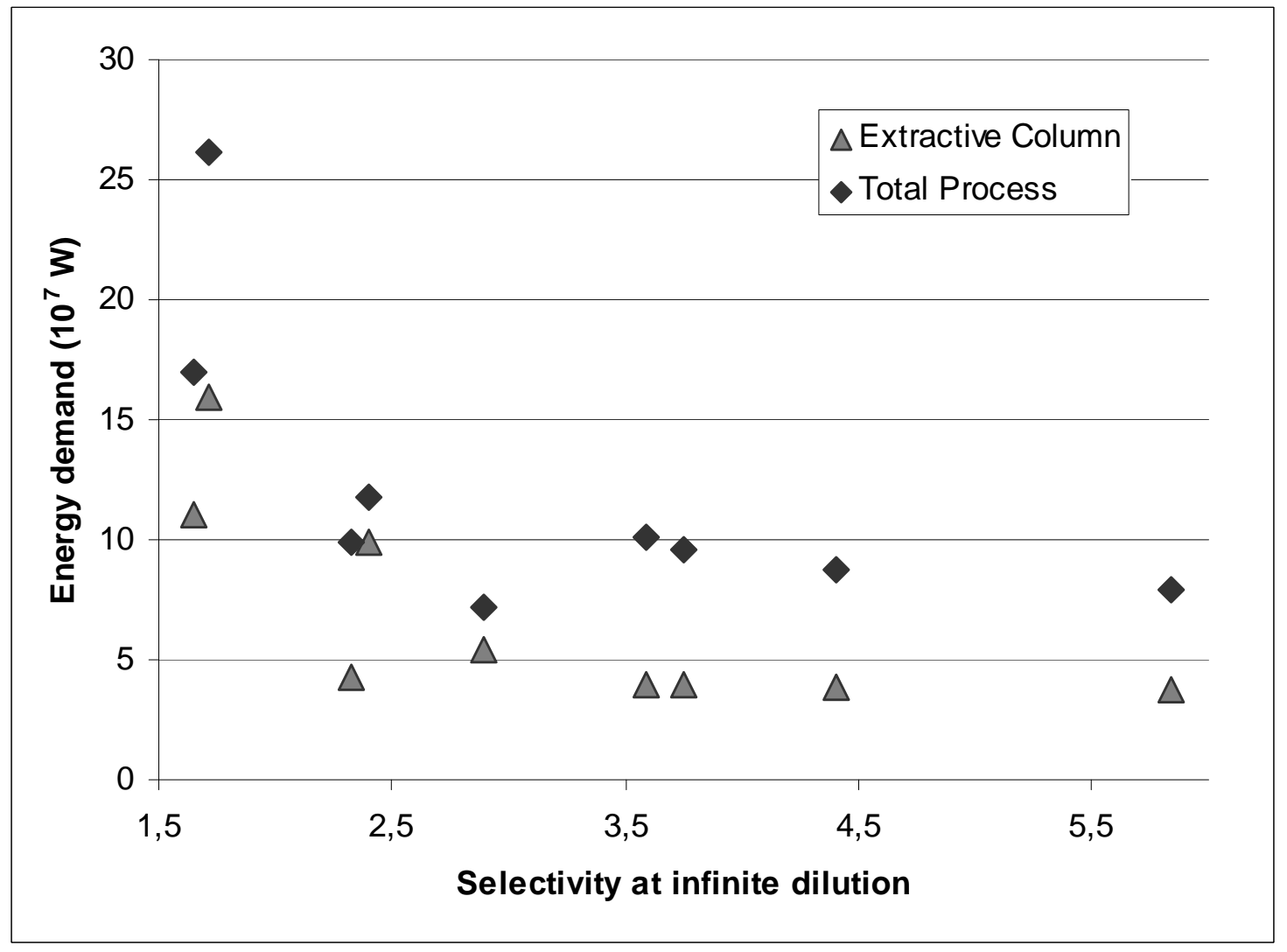

Figure 5: Energy demand of the extractive column and of the total process as a function of selectivity.

The selectivity is a useful but not a very accurate analysis method for the effectiveness of an extractive distillation process. This is illustrated in Fig. 5, where the energy demand of the extractive column and of the total extractive distillation process after the flowsheet optimization is plotted as a function of the selectivity $S_{i j}^{\infty}$. Interestingly, the selectivity at infinite dilution gives very poor predictions for the process energy demand for low values (e.g. water and p-xylene at $S_{i j}^{\infty} \approx 2.4$ ). For higher values of selectivity the energy demand does not change significantly.

Because of their higher reliability, the results obtained with the RBM are used here to screen the different entrainers. DMSO and water (since it is an environmentally friendly substance) are the only candidates yielding acetone as the product of the extractive distillation column which are selected for further consideration in the next step. Ethanol and isopropanol are discarded because of the high minimum energy demand of the process. In case of methanol as the distillate product, the differences in process energy demand are not as pronounced and the decision of the cut-off point is harder to define. We choose chlorobenzene, p-xylene (UNIFAC/UNIQUAC) and mesitylene for further evaluation. For these five 
different entrainer choices the optimal number of stages, the optimal entrainer flowrate and the optimal reflux ratios of the two columns are calculated by MINLP optimization.

It should be noted that all entrainer candidates listed above (with the obvious exception of water) are harmful substances. The case study is nevertheless meaningful since we will then know what properties we should look for (the same mixture behaviour as DMSO but without its toxicity) in further CAMD steps.

\section{MINLP optimization of the extractive flowsheet}

\subsection{MINLP optimization algorithm}

The shortcut calculations with the RBM cannot provide any information about the optimal number of trays and the optimal feed tray location of the distillation columns as the minimum energy demand is calculated for columns with an infinite number of stages. For a more detailed column design an economic trade-off has to be found between investment cost (mainly determined by the column diameter and number of trays) and operating cost (defined by the energy duty of the columns). Since the number of trays and the feed tray locations are discrete variables while the energy duties, flow-rates and compositions are continuous variables, MINLP optimization is usually used for identifying the optimal column designs and the process operating point.

However, robustness and convergence of complex MINLP problems to good local optima still remain issues that need to be addressed for an efficient column optimization, especially when the entire extractive flowsheet consisting of two columns and a recycle stream (cf. Fig.1) has to be optimized. To overcome these difficulties a novel solution approach has been developed, where a sequence of relaxed MINLP problems with decreasing bounds are solved.

This solution algorithm as well as the column model are described in our previous publications $(14 ; 15)$, therefore only a brief summary is given here. The main idea is to replace the integer variables by continuous decision variables. To ensure an integer solution, special integrality constraints are added to force the continuous decision variables to discrete values (21). These integrality constraints are relaxed and the resulting relaxed MINLP is solved in a series of successive optimization runs with decreasing relaxation bounds. The solution procedure is thus termed SR-MINLP (successive relaxed MINLP).

The integer decision variables of a distillation column are the total number of trays and the location of the feed tray. As already observed by Viswanathan and Grossmann (22) and confirmed by our own experience (14), the distribution of a single column feed for a simple distillation column is optimal when the entire feed is introduced on a single stage, i.e. the optimal solution of the relaxed problem will usually yield an integer solution for the feed stage. This, however, is not true for extractive columns. Here, a distributed entrainer feed seems to be optimal and therefore integrality constraints have to be introduced (15) for the feed location in this case.

The reboiler or condenser location are equally forced to integer values by successively tightened integrality constraints. After reformulation, the MINLP optimization problem only contains continuous variables and thus a computational costly Branch and Bound tree search or an Outer Approximation iteration to handle binary variables can be avoided. The continuous model therefore exhibits shorter solution times as an equivalent MINLP model. 
In case of this large optimization problem the advantage of the continuous model is even more pronounced than for the single column case. The combinatorial complexity of the solution of the MINLP model rises exponentially when multiple columns with discrete decisions are combined in a flowsheet optimization. In contrast, the combinatorial effort is significantly reduced when the optimization of column sequences is carried out with continuous optimization models (15).

\subsection{Initialization of the MINLP problem}

A key feature of the proposed synthesis framework is that the results from the RBM shortcut screening can be used for an excellent initialization of the MINLP problem $(13 ; 14)$. The entrainer flowrate determined from flowsheet optimization using shortcut models (cf. Section 4.1.3) and the resulting reboiler and condenser energies provide excellent initial values for the MINLP optimization. Furthermore, approximate column profiles can be determined from the pinch points calculated by the RBM, which allows the initialization of tray composition and temperatures very close to their final values. The information on flow rates, compositions, temperatures and minimal energy demands from the shortcut step can also be used to provide tight bounds for the optimization problem.

This excellent initialization together with the new solution algorithm outlined above has helped robustness, reliability and efficiency of the MINLP optimization dramatically. A typical optimization problem consist of about 350 discrete decision variables, more than 5000 continuous variables and the underlying highly nonlinear thermodynamic models. Still, solution times are less than 10 minutes with the NLP solver SNOPT 6.2-1 on a workstation with a $3.06 \mathrm{GHz} \mathrm{CPU}$ and $3.75 \mathrm{~GB}$ RAM (15). Thus, all entrainer candidates could be compared by MINLP optimization. Since the shortcut calculations precede the rigorous optimization, it is suggested to only optimize the flowsheets for the most promising entrainer candidates to further reduce the effort in the MINLP optimization step.

\subsection{MINLP optimization of the example flowsheets}

MINLP problems as described in Sections 5.1 and 5.2 for the rigorous optimization of the extractive distillation process shown in Fig. 1 were formulated and solved for various entrainer choices. Only the efficient entrainers DMSO and chlorobenzene and the environmental benign entrainer water need to be evaluated in the rigorous optimization step due to the ranking of the entrainer alternatives by the minimum process energy demand with the RBM in the preceding step of the synthesis framework (cf. Tab. 4). However, we have also included the evaluation of the entrainers mesitylene and p-xylene (UNIFAC and UNIQUAC) in the rigorous optimization step for a more comprehensive comparison of the results.

All product purities were set to values larger than $99.5 \mathrm{~mol} \%$ except for the cases where methanol is the distillate product of the entrainer recovery column: here, the methanol purities were set to $98.2 \mathrm{~mol} \%$ and $98.9 \mathrm{~mol} \%$ for the entrainers water and DMSO, respectively, since higher purities could not be obtained. Optimal values are found for the number of column trays and the feed tray locations (discrete values) as well as for the reboiler and condenser duties and for the recycle flowrate and composition (continuous values). The exact formulation of the economic objective function accounting for operating and investment 
cost can be found in (14). The rigorous optimization results together with the entrainer flowrate and minimum energy demand from the shortcut evaluation with the RBM are displayed in Tables 5 and 6.

\begin{tabular}{|l|c|c|c|c|c|}
\hline \multicolumn{5}{|c|}{ distillate acetone } \\
\hline & \multicolumn{2}{|c|}{ RBM } & \multicolumn{3}{c|}{ MINLP } \\
\hline $\begin{array}{l}\text { entrainer } \\
\text { candidate }\end{array}$ & $E_{R B M} / F$ & $Q_{B, \text { min }} / F$ & $E_{\text {opt }} / F$ & $\begin{array}{c}Q_{B, \text { opt }} / F \\
\text { total process }\end{array}$ & $\begin{array}{c}\text { Cost/F } \\
\text { total process }\end{array}$ \\
\hline DMSO & 0.771 & 7.200 & 0.992 & 7.57 & 34.20 \\
\hline water & 0.933 & 11.803 & 1.886 & 11.11 & 44.64 \\
\hline \multicolumn{5}{|c|}{ distillate methanol } \\
\hline chlorobenzene & 2.02 & 7.93 & 2.382 & 8.37 & 32.56 \\
\hline p-xylene (UNIFAC) & 1.67 & 8.71 & 2.249 & 9.33 & 35.62 \\
\hline mesitylene & 1.55 & 9.56 & 1.900 & 10.38 & 38.92 \\
\hline p-xylene (UNIQUAC) & 2.38 & 9.94 & 2.597 & 10.63 & 39.67 \\
\hline
\end{tabular}

Table 5: Results for entrainer flowrate, process reboiler energy demand and process cost for different entrainer alternatives obtained with the rigorous MINLP optimization in comparison with values from the RBM shortcut calculations. Process costs include investment and operating costs. Energies are given in $10^{7} \mathrm{~W} / \mathrm{kmol}$ and costs in $10^{6} € / \mathrm{kmol}$.

\begin{tabular}{|l|c|c|c|c|c|}
\hline \multicolumn{4}{|c|}{ distillate acetone } \\
\hline & \multicolumn{2}{|c|}{ Number of Trays } & \multicolumn{3}{c|}{ Feed Tray } \\
\hline entrainer candidate & extr. column & recycle column & extr. E & extr. F & recycle F \\
\hline DMSO & 35 & 13 & 2 & 27 & 5 \\
\hline water & 54 & 26 & 33 & 50 & 15 \\
\hline \multicolumn{5}{|c|}{ distillate methanol } \\
\hline chlorobenzene & 42 & 16 & 10 & 26 & 7 \\
\hline p-xylene (UNIFAC) & 43 & 13 & 10 & 24 & 6 \\
\hline mesitylene & 37 & 9 & 4 & 22 & 4 \\
\hline p-xylene (UNIQUAC) & 47 & 11 & 8 & 30 & 6 \\
\hline
\end{tabular}

Table 6: Results for the optimal number of trays and feed tray locations for the different entrainer alternatives obtained with MINLP optimization.

When comparing the results of the shortcut evaluation with the RBM and the MIMLP optimization results, the order of the entrainer candidates in the ranking of economic efficiency is not changed except for DMSO, which turns out to be more expensive than chlorobenzene due to higher energy costs. These are due to the high boiling point of DMSO $\left(190.7^{\circ} \mathrm{C}\right)$ is well above the boiling points of the other entrainer candidates. Consequently, steam at a higher pressure level is required for the reboilers of both columns when DMSO is the entrainer choice, raising the cost of energy in this case. The energy demand of the rigorously optimized processes is well correlated to the minimal energy demand predicted by the shortcut evaluation: the ratios of minimal to optimized energy demands range from 0.92 for mesitylene to 0.95 for DMSO. The exception is water, where a lower product purity for 
methanol and a significantly higher entrainer flowrate than the minimal flowrate give rise to a lower optimized energy demand than the minimal energy demand from the shortcut evaluation. Still, the results demonstrate that the assumption of infinitely large columns for the shortcut calculations is a very reasonable simplification, since the optimized energy demand is close to the minimal energy demand for all entrainer alternatives. The ratios of the minimal entrainer flowrates to the optimized entrainer flowrates are between 0.78 for DMSO and 0.92 for p-xylene (UNIQUAC), again with the exception of water with a ratio of 0.5 .

The significance of the rigorous optimization step is emphasized when the optimal values for the tray numbers and feed tray locations for the different entrainer candidates are compared. Here, a noticeable variance in the optimal values for the different entrainers is observed, especially for the entrainer feed location.

\subsection{Comparison of the different screening methods with the MINLP results}

When the results from MINLP optimization are taken as the "true" process cost, the different proposed guidelines for entrainer selection can be reviewed and compared with respect to their prediction accuracy.

\subsubsection{Comparison of the MINLP results with the selectivity criterion}

The total annualized cost (TAC) as determined from MINLP optimization as a function of the selectivity $\left(S_{i j}^{\infty}\right)$ is plotted in Fig. 6. As can be seen in the figure and also deduced from the low regression coefficient $R^{2}=0.4911$, the prediction of the total process cost is not possible from selectivity alone. These findings further support the results already discussed in Sections 4.1.1 and 4.2.2. Selectivity alone should therefore not be used as the only criterion for the screening of entrainer candidates.

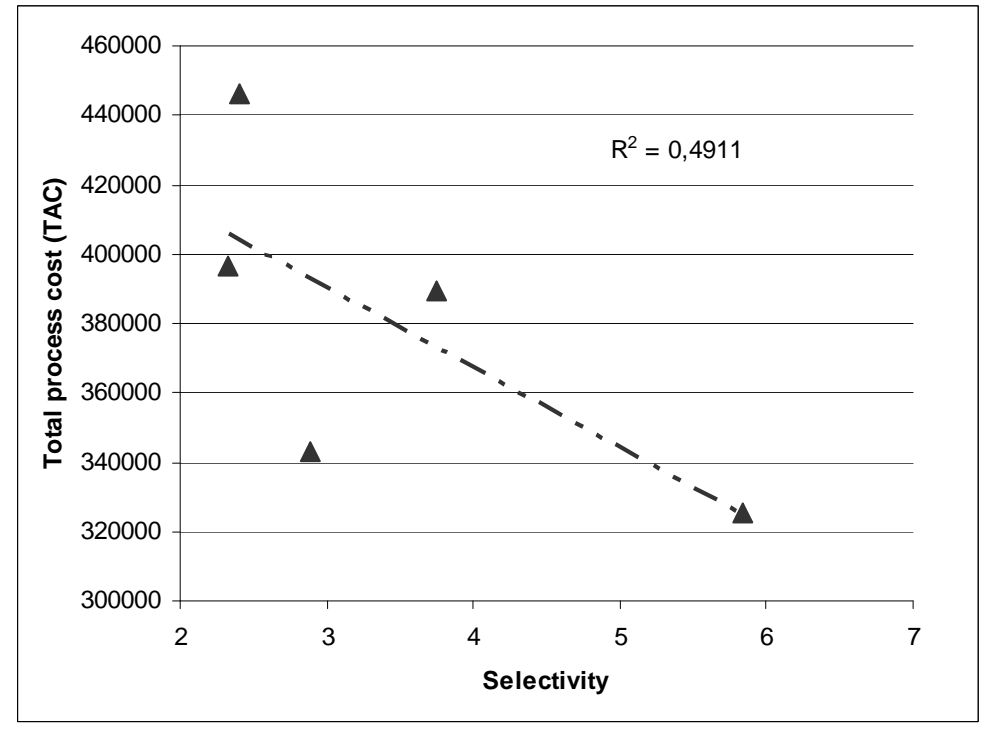

Figure 6: Total annualized cost of the extractive process (MINLP results) as a function of selectivity. 


\subsubsection{Comparison of the MINLP results with the capacity selectivity criterion}

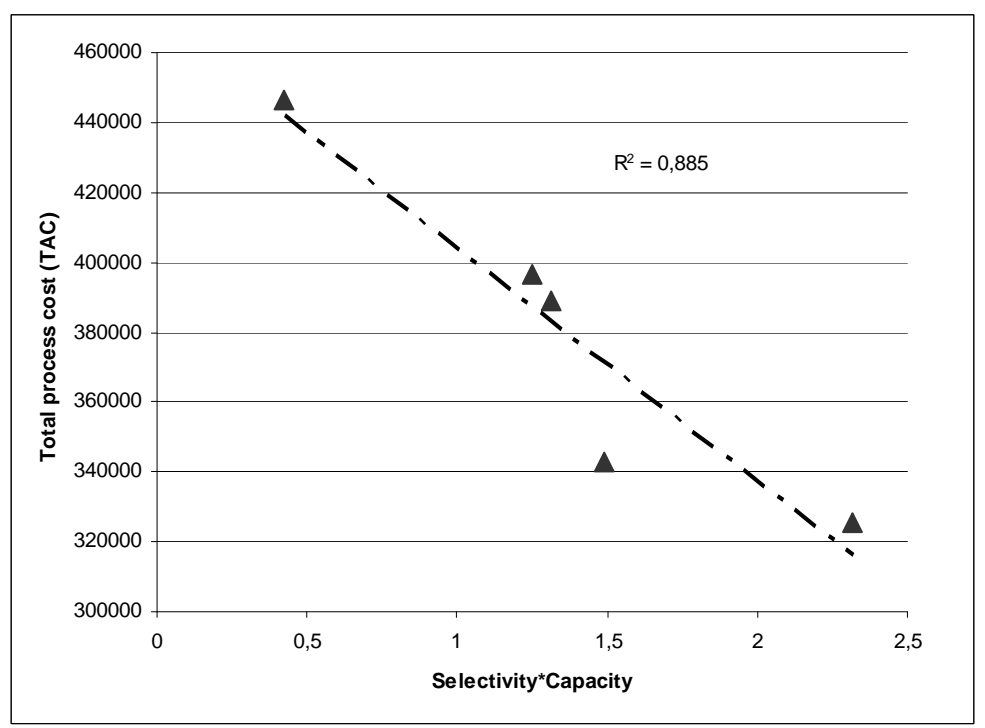

Figure 7: Total annualized cost of the extractive process (MINLP results) as a function of capacity.selectivity $\left(C_{j, \text { Entrainer }}^{\infty} \cdot S_{i j}^{\infty}\right)$.

Another criterion mentioned for entrainer selection from literature (9) suggests to evaluate

$$
C_{j, \text { Entrainer }}^{\infty} \cdot S_{i j}^{\infty} \text {. }
$$

This equation takes the selectivity and the capacity of an entrainer into consideration. The $T A C$ is shown in Fig. 7 as a function of $C_{j, \text { Entrainer }}^{\infty} \cdot S_{i j}^{\infty}$. It can be seen from the graph and from $R^{2}=(0.885)$ that the prediction accuracy of this screening heuristic is already better than a ranking based on selectivity alone.

\subsubsection{Comparison of the MINLP results with the RBM}

Finally the results from the MINLP optimization as a function of the minimum energy demand as determined with the RBM are shown in Fig. 8. The prediction accuracy is already quite good as can be seen in the graph and in the even higher value of $R^{2}=0.9278$. Note that the $R^{2}$ value climbs to 0.9854 when the entrainer DMSO is excluded since the TAC for DMSO is dependent on the specified steam cost for the high pressure steam that is required for the reboiling of the DMSO at its high boiling point (cf. Section 5.3).

From the results of this case study we can therefore conclude that the RBM gives a very good estimate of the total process cost. Since the results of the RBM can be used to initialize the MINLP optimization, it is recommended to use the RBM as a screening tool for entrainer candidates. When the evaluation of the RBM is not practical or not available, the expression $C_{j, \text { Entrainer }}^{\infty} \cdot S_{i j}^{\infty}$ can be used. It should be noted that this expression can only serve as a rule of thumb and is limited to the screening of entrainer alternatives since minimum entrainer flowrates and reflux bounds are not available. The selectivity alone, however, is not a very good screening tool and should not be used alone to predict entrainer performance. 


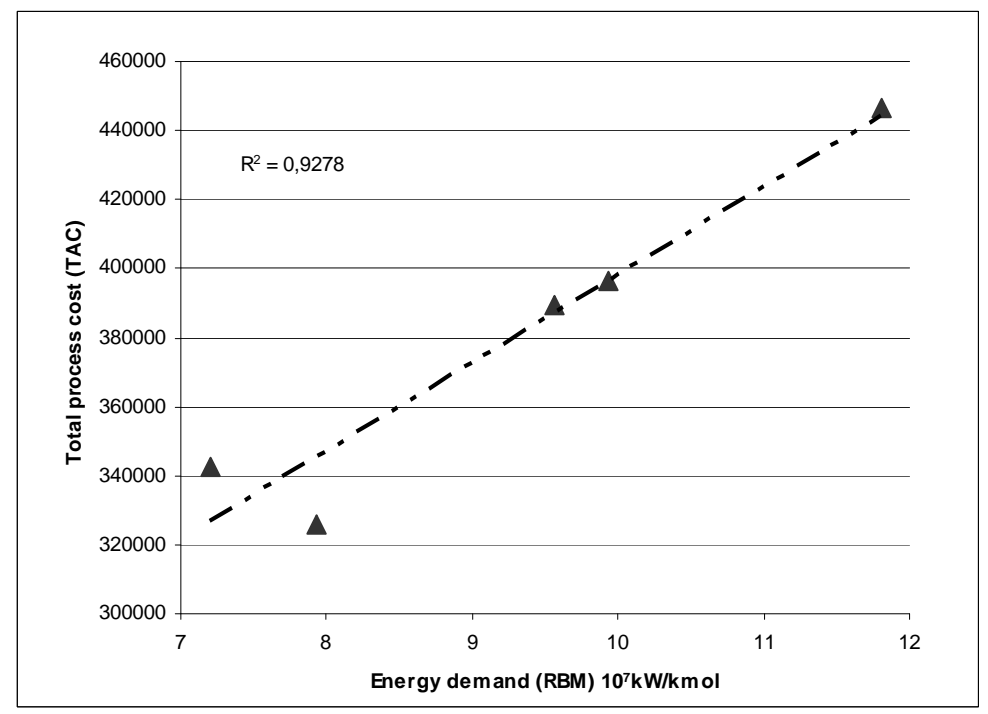

Figure 8: Total annualized cost of the extractive process (MINLP results) as a function of the energy demand as calculated with the RBM.

\section{Summary and conclusion}

In this paper, a short summary of the generation and evaluation of different entrainers for extractive distillation is given. It is shown that possible entrainer candidates can be obtained from heuristics, literature studies or the more elaborate methods available through CAMD.

It is shown that the different methods for entrainer evaluation have their strengths and weaknesses. The selectivity gives a first estimate for a relative ranking of entrainer alternatives for the same flowsheet structure and for the same type of splits. As soon as this structure is changed, the results cannot be easily compared to other cases. Furthermore, a simple correlation of selectivity to TAC or minimum energy demand is not possible (cf. Fig. 4 and 5). The use of the product of capacity and selectivity already improves the screening accuracy; however, no details on the operating point of the extractive column are available from this method (cf. Section 5.4).

The Rectification Body Method (RBM) allows a comparison of different flowsheet structures as well as a comparison of different entrainer alternatives. This information comes at a slightly higher computational cost. It allows, however, a very accurate ranking of the generated entrainers and the information generated with this mixture, especially the determination of the optimum entrainer flowrate, can be used for an excellent initialization of a detailed MINLP optimization.

This MINLP optimization is the final step of the proposed synthesis framework for extractive distillation. Here the first two to three optimal entrainer choices as determined with the RBM are evaluated further. In this case the choices would be DMSO and chlorobenzene (although water will probably be preferred over the other options since it is environmentally friendly and induces only a moderate economic penalty). The combination of the tools CAMD, RBM and MINLP optimization is therefore very well suited for the systematic design of an optimal extractive distillation process.

This approach will be extended to other flowsheets with recycle streams (much like the 
ternary pressure swing process (13)) in the future. Another aspect that will be integrated into our framework is energy integration, which might offer significant cost-savings for coupled processes.

\section{Acknowledgements}

This work was funded by the Deutsche Forschungsgemeinschaft (DFG) under grant MA 1188/26-1.

\section{References}

[1] ASPEN Technology (2004) ASPEN Technology, Inc., Cambridge.

[2] Bausa, J., von Watzdorf, R., and Marquardt W. (1998), AIChE Journal, 44, 21812198.

[3] Blass, E., Entwicklung verfahrenstechnischer Prozesse, Salle \& Sauerländer, Frankfurt am Main (1989).

[4] Brüggemann, S. and Marquardt, W. (2004) AIChE Journal, 50, 1129-1149.

[5] Doherty, M.F. and Malone, M.F., Conceptual Design of Distillation Systems, McGraw-Hill, New York (2001).

[6] Gani, R.,Achenie, L.E.K., and Venkatasubramanian, V., Introduction to CAMD. In: Achenie, L.E.K., R. Gani and V. Venkatasubramanian: Computer Aided Molecular Design: Theory and Practice, Elsevier, Amsterdam, (2003).

[7] Harper, P.M., A multi-phase, multi-level framework for computer aided molecular design, Technical University of Denmark, Lyngy, (2000).

[8] Harper, P.M. and Gani, R. (2000) Computers and Chemical Engineering, 24, 677683.

[9] Jork, C., Kristen, C., Pieraccini, D., Stark, A., Chiappe, C., Beste, Y.A., and Arlt, W. (2005) Journal of Chemical Thermodynamics, 37, 537-558.

[10] Karunanith, A.T., Achenie, L.E.K, and Gani, R. (2005) Industrial and Engineering Chemistry Research, 44, 4785-4797.

[11] Knapp, J.P., and Doherty, M.F. (1989) Industrial and Engineering Chemistry Research, 28, 564-572.

[12] Knapp, J.P., and Doherty, M.F. (1990) AIChE Journal, 36, 969-983.

[13] Kossack, S., Kraemer, K. and Marquardt, W., In: Sorensen E. (Ed.): Distillation \& Absorption 2006, IChemE, London, (2006).

[14] Kossack, S., Kraemer, K. and Marquardt, W. (2006) Industrial and Engineering Chemistry Research, 45, 8492-8502. 
[15] Kraemer, K., Kossack, S., and Marquardt, W., In: V. Plesu, P.S. Agachi (Eds.): Proceedings of the 17th European Symposium On Computer Aided Process Engineering - ESCAPE 17, Bucharest, (2007).

[16] Laroche, L., Bekiaris, N., Andersen, H.W., and Morari, M. (1991) Canadian Journal of Chemical Engineering, 69, 1302-1319.

[17] Laroche, L., Bekiaris, N., Andersen, H.W., and Morari, M. (1992) AIChE Journal, 38, 1309-1328.

[18] Lei, Z., Li C. and Chen, B., (2003) Separation and Purification Reviews, 32, 121-213.

[19] Momoh, S.O. (1991) Separation Science and Technology, 26, 729-742.

[20] Rowley, R.L., Wilding, W.V., Oscarson, J.L., Yang, Y., Zundel, N.A., Daubert, T.E., and Danner, R.P., DIPPR Data Compilation of Pure Chemical Properties, Design Institute for Physical Properties, AIChE, New York (2006).

[21] Stein, O., Oldenburg, J., and Marquardt, W., (2004) Computers and Chemical Engineering, 28, 1951-1966.

[22] Viswanathan, J., and Grossmann, I., (1993) Computers and Chemical Engineering, 17, 949-955. 